\title{
Los inhibidores de la bomba de protones no disminuirían el sangrado por anticoagulantes 0 aspirina
}

\author{
Proton pump inhibitors would not decrease bleeding due to anticoagulants or aspirin
}

\section{Comentado de:}

Moayyedi $P$ et al. Gastroenterology. 2019 Aug;157(2):403412.e5

\section{Objetivo}

Evaluar si la terapia con inhibidores de la bomba de protones (IBP) podría reducir el riesgo del sangrado gastrointestinal superior por antiplaquetarios y anticoagulantes.

\section{Diseño, lugar y participantes}

Ensayo clínico aleatorizado, multicéntrico, doble ciego. Incluyó 17.598 participantes con enfermedad cardiovascular estable y enfermedad arterial periférica, en 580 centros de 33 países.

\section{Intervención}

Los participantes fueron asignados en forma aleatoria a dos grupos: pantoprazol $40 \mathrm{mg}$ diarios o placebo. A su vez, los pacientes fueron también aleatorizados a recibir: rivaroxabán $2.5 \mathrm{mg}$ dos veces al día con aspirina $100 \mathrm{mg}$ una vez al día, rivaroxabán $5 \mathrm{mg}$ dos veces al día o aspirina $100 \mathrm{mg}$ solamente. La parte del estudio que evaluó el efecto deaspirina y rivaroxaban fue interrumpida precozmente (a los 2 años), sin embargo la parte que evaluó pantoprazol continuó durante 3 años.

\section{Medición de resultados principales}

El resultado primario fue el tiempo hasta el primer evento gastrointestinal superior, definido como un resultado compuesto de hemorragia manifiesta, hemorragia gastrointestinal superior de una lesión gastroduodenal o de origen desconocido, hemorragia oculta, úlcera gastroduodenal sintomática o 5 erosiones, obstrucción gastrointestinal superior o perforación.

\section{Resultados principales}

No hubo diferencias significativas en los eventos gastrointestinales superiores entre el grupo de pantoprazol (102 pacientes tuvieron algún evento, de un total de 8791 asignados a esa rama) y el grupo de placebo (116 de 8807) (Riesgo relativo[RR], 0,88; Intervalo de Confianza [IC] $95 \%$ 0,67 a 1,15). Desglosando el desenlace principal compuesto, el pantoprazol redujo significativamente el sangrado de las lesiones gastroduodenales (RR, 0,52 IC $95 \%, 0,28$ a 0,94) (ver Tabla 1).

Tabla 1. Eficacia del omeprazol para prevenir eventos adversos gastrointestinales asociados con fármacos antiplaquetarios y anticoagulantes

\begin{tabular}{|l|c|c|c|}
\hline Evento & $\begin{array}{c}\text { Pantoprazol } \\
\%\end{array}$ & $\begin{array}{c}\text { Placebo } \\
\%\end{array}$ & P valor \\
\hline Sangrado de ulcera (diagnóstico por endoscopía) & 0,2 & 0,4 & 0,03 \\
Sangrado de origen desconocido & 0,6 & 0,5 & 0,68 \\
Sangrado presunto por descenso de hemoglobina & 0,1 & 0,1 & 0,99 \\
Úlcera sintomática & $<0,1$ & 0,2 & 0,07 \\
Dolor gastrointestinales con múltiples ulceraciones & $<0,1$ & $<0,1$ & 0,37 \\
Obstrucción o perforación gastrointestinal superior & 0,2 & 0,2 & 0,41 \\
\hline
\end{tabular}

\section{Conclusiones}

En este ensayo clínico se encontró que el uso rutinario de IBP en pacientes con dosis bajas de anticoagulantes $y / 0$ aspirina para enfermedad cardiovascular estable no reduce los eventos gastrointestinales superiores, pero pue- den reducir el sangrado de las lesiones gastroduodenales.

Fuente de financiamiento/Conflicto de interés de los autores: El estudio fue financiado por Bayer AG. Los autores principales tienen extensos vínculos con la industria farmacéutica.

\section{Comentario}

Este ensayo clínico de alta calidad y con gran tamaño muestral ha documentado que el uso rutinario de pantoprazol no redujo el sangrado gastrointestinal en pacientes con aspirina y/o rivaroxaban.

Hasta ahora, las guías de práctica clínica no habían emitido una recomendación sobre el uso de IBP en estos casos ${ }^{2}$ Considerando que existía cierta duda sobre su eficacia, y el uso de IBP suele estar englobado bajo el eslogan de "protector gástrico" ${ }^{3}$, constituye una clase de fármacos ampliamente prescrita y, habitualmente, de manera inadecuada ${ }^{4}$. 
Si bien el énfasis del estudio está puesto en los sangrados gastrointestinales, el uso de aspirina y anticoagulantes también se asocia a un mayor riesgo de sangrado cerebral ${ }^{5}$. También es importante destacar las preocupaciones sobre el uso a largo plazo de IBP y la aparición de otros efectos adversos: infecciones intestinales, hipergastrinemia (con dificultad para su deprescripción), insuficiencia renal, déficit de vitamina B12 y riesgo de fractura, entre otros ${ }^{6}$.

\section{Conclusiones del comentador}

Dado que no existiría un beneficio a nivel gastrointestinal con el uso de IBP en pacientes que consumen aspirina o anticoagulantes, el profesional de la salud debería hacer una cuidadosa valoración del riesgo de sangrado gastrointestinal de cada paciente y restringir su uso a casos explícitamente justificados, considerando que existen más certezas sobre los potenciales daños que sobre los beneficios del uso de los IBP a largo plazo.

Juan Victor Ariel Franco [ Servicio de Medicina Familiar y Comunitaria, Hospital Italiano de Buenos Aires, Departamento de Investigación, Instituto Universitario Hospital Italiano juan.franco@hospitalitaliano.org.ar ]

Franco JVA Los inhibidores de la bomba de protones no disminuirían el sangrado por anticoagulantes o aspirina. Evid Actual Pract Ambul. 2019;1(2):e002011. Comentado de: Moayyedi P et al. Pantoprazole to Prevent Gastroduodenal Events in Patients Receiving Rivaroxaban and/or Aspirin in a Randomized, Double-Blind, Placebo-Controlled Trial. JGastroenterology. 2019 Aug;157(2):403-412.e5

\section{Referencias}

1. Moayyedi P, Eikelboom JW, Bosch J, Connolly SJ, Dyal L, Shestakovska O. Pantoprazole to Prevent Gastroduodenal Events in Patients Receiving Rivaroxaban and/or Aspirin in a Randomized, Double-Blind, Placebo-Controlled Trial. Gastroenterology. 2019;157(2):31054846-31054846. Available from: 10.1053/j.gastro.2019.04.041.

2. January CT, Wann LS, Calkins H, Chen LY, Cigarroa JE, Jr JC, et al. 2019 AHA/ACC/HRS Focused Update of the 2014 AHA/ACC/HRS Guideline for the Management of Patients With Atrial Fibrillation: A Report of the American College of Cardiology/American Heart Association Task Force on Clinical Practice Guidelines and the Heart Rhythm Society. J Am Coll Cardiol. 2019;9(74):104-132. Available from: 10.1016/j.jacc.2019.01.011.

3. Domingo JJS. Las consecuencias del consumo inadecuado de omeprazol; 2018. Available from: https://elpais.com/elpais/2018/02/02/ciencia/ 1517569615_996884.html [Last access: 2019-08-19].

4. Haastrup PF, Thompson W, Søndergaard J, Jarbøl DE. Side Effects of Long-Term Proton Pump Inhibitor Use: A Review. Basic Clin Pharmacol Toxicol. 2018;123(2):114-121. Available from: 10.1111/bcpt.13023.

5. Charidimou A, Karayiannis C, Song TJ, Orken DN, Thijs V, Lemmens R, et al. Brain microbleeds, anticoagulation, and hemorrhage risk: Meta-analysis in stroke patients with AF. Neurology. 2017;89(23):2317-2326. Available from: 10.1212/WNL.0000000000004704.

6. Ribiere S, Guillaumot MA, Barré A, Ali EA, Barret M, Chaussade S, et al. [What is the REAL long-term risk of proton pump inhibitors?]. Presse medicale (Paris, France : 1983). 2019 may;48(5):503-510. Available from: 10.1016/j.lpm.2019.02.011. 